\title{
Enhanced cancer stem cell properties of a mitotically quiescent subpopulation of p75NTR-positive cells in esophageal squamous cell carcinoma
}

\author{
HIROFUMI KOJIMA $^{1}$, TOMOYUKI OKUMURA ${ }^{1}$, TETSUJI YAMAGUCHI ${ }^{1}$, \\ TAKESHI MIWA $^{1}$, YUTAKA SHIMADA ${ }^{2}$ and TAKUYA NAGATA ${ }^{1}$
}

\begin{abstract}
${ }^{1}$ Department of Surgery and Science, Graduate School of Medicine and Pharmaceutical Sciences, University of Toyama, Toyama city, Toyama 930-0194; ${ }^{2}$ Department of Nanobio Drug Discovery, Graduate School of Pharmaceutical Sciences, Kyoto University, Sakyo-ku, Kyoto 606-8501, Japan
\end{abstract}

Received February 16, 2017; Accepted April 27, 2017

DOI: 10.3892/ijo.2017.4001

\begin{abstract}
Mitotically quiescent cancer stem cells (CSCs) possess higher malignant potential than other CSCs, indicating their higher contribution to therapeutic resistance than that of other CSCs. In esophageal squamous cell carcinoma (ESCC), p75 neurotrophin receptor (p75NTR) is expressed in a candidate CSC population showing high tumorigenicity and chemoresistance. In the present study, we isolated and characterized quiescent CSCs population in ESCC based on p75NTR expression and cell cycle status. Expression of p75NTR and Ki-67 in ESCC cell lines (KYSE cells) and surgically resected ESCC specimens was detected by performing immunocytochemical analysis. p75NTR-positive KYSE cells were fractionated into quiescent and proliferating cells by performing flow cytometry with a fluorescent DNA-staining dye to determine their CSC phenotype. Immunocytochemical analysis showed that 21.8 and $36.5 \%$ of the p75NTR-positive cells were $\mathrm{Ki}$-67-negative (G0), which accounted for 11.4 and $15.7 \%$ of cells in KYSE-30 and KYSE-140 cell lines, respectively. Flow cytometric cell sorting showed that p75NTR-positive cells in the G0-G1 phase (p75NTR-positive/ G0-1 cells) but not in the S-G2-M phase (p75NTR-positive/SG2-M cells) showed strong expression of stem cell-related genes Nanog, BMI-1, and p63; high colony formation ability; high tumorigenicity in a mouse xenograft model; and strong chemoresistance against cisplatin because of the expression of drug resistance genes ABCG2 and ERCC1. Label-retention
\end{abstract}

Correspondence to: Dr Tomoyuki Okumura, Department of Surgery and Science, Graduate School of Medicine and Pharmaceutical Sciences, University of Toyama, 2630 Sugitani, Toyama City, Toyama 930-0194 Japan

E-mail: okumura@med.u-toyama.ac.jp

Key words: esophageal cancer, p75 neurotrophin receptor, CD271, quiescent cancer stem cell, cell cycle assay showed that $3.4 \%$ p75NTR-positive cells retained fluorescent cell-tracing dye, but p75NTR-negative cells did not. Immunohistochemical analysis of ESCC specimens showed p75NTR expression in 39 of 95 (41.1\%) patients, with a median of $13.2 \%$ (range, 3.0-80.1\%) p75NTR-positive/Ki-67-negative cells, which were found to be associated with poorly differentiated histology. Our results suggest that p75NTR-positive/ G0-1 cells represent quiescent CSCs in ESCC and indicate that these cells can be used as targets to investigate molecular processes regulating CSC phenotype and to develop novel therapeutic strategies.

\section{Introduction}

Esophageal squamous cell carcinoma (ESCC) is one of the most common malignancies worldwide (1). Despite recent progress in multidisciplinary therapy and diagnostic approach, prognosis of patients with ESCC remains poor because of the high incidence of metastasis and therapeutic resistance $(2,3)$. However, increased understanding of the biological basis of ESCC has led to the development of improved therapeutic and diagnostic strategies.

Cancer stem cells (CSCs) are small number of cells with stem cell properties such as self-renewal, differentiation potential, tumorigenicity and therapeutic resistance (4-8). Recent studies have detected mitotically quiescent CSCs in solid tumors such as melanomas and breast and pancreatic cancers (9-11). CSCs are predominantly present in the resting phase of the cell cycle and undergo proliferation in response to external stimuli (12). Quiescent CSCs possess higher tumorigenicity and chemoresistance than other CSCs, indicating that they are more responsible for the malignant potential of tumors and they can be more effective therapeutic targets (10-14).

In ESCC, p75 neurotrophin receptor (p75NTR, CD271) is expressed in a candidate CSC population that shows strong expression of stem cell-related genes, high sphere/colony formation ability, high tumorigenicity in a mouse xenograft model and chemoresistance (15-18). Originally, p75NTR was found to be expressed in the stem cell fraction of normal esophageal keratinocytes, most of which were in the resting 
phase of the cell cycle $(19,20)$. Cell proliferation marker Ki-67 expression analysis and flow cytometric cell cycle analysis showed that $<50 \%$ p75NTR-positive cells maintain a mitotically quiescent phenotype in ESCC (18). However, isolation and characterization of these quiescent cells from p75NTRpositive cells has not been performed to date. In the present study, we isolated and characterized this candidate quiescent CSC population in ESCC based on p75NTR expression and cell cycle status.

\section{Materials and methods}

Cell culture. Human ESCC cell lines KYSE-30 and KYSE-140 were established by Shimada et al (21) and were cultured in a T75 tissue culture flask (Thermo Fisher Scientific, Inc., Yokohama, Japan) containing DMEM/Ham's F-12 medium (Wako Pure Chemical Industries, Ltd., Osaka, Japan) supplemented with 5\% fetal calf serum (FCS; Gibco, Grand Island, NY, USA) and 1\% 100X antibiotic-antimycotic (Gibco/Thermo Fisher Scientific, Waltham, MA, USA) by using a standard previously reported method. The cells were maintained at $37^{\circ} \mathrm{C}$ in a humidified atmosphere of $5 \% \mathrm{CO}_{2}$ until confluence.

Cell sorting based on p75NTR expression and cell cycle status. Cultured cells were washed once with phosphatebuffered saline (PBS), then dissociated from culture plates by using $0.25 \%$ trypsin EDTA $(1 \mathrm{X})$ and phenol red (Life Technologies, Carlsbad, CA, USA) and were centrifuged at $300 \mathrm{x}$ g for $10 \mathrm{~min}$. Single cells were resuspended in PBS containing 2\% FCS and allophycocyanin (APC)-conjugated human CD271 (LNGFR) antibody (Miltenyi Biotec GmbH, Bergisch Gladbach, Germany) or a compared isotype control were incubated in the dark at $4^{\circ} \mathrm{C}$ for $30 \mathrm{~min}$. After washing twice with PBS containing 2\% FCS, the cells were resuspended in Hank's balanced salt solution (Wako Pure Chemical Industries), were treated with Vybrant ${ }^{\circledR}$ DyeCycle ${ }^{\mathrm{TM}}$ Violet stain (DCV; Invitrogen/Molecular Probes, Eugene, OR, USA) and were mixed well. Next, the cells were incubated at $37^{\circ} \mathrm{C}$ for $30 \mathrm{~min}$, protected from light. Cell samples by using a flow cytometer (BD FACSAria ${ }^{\mathrm{TM}}$ II; BD Biosciences, San Jose, CA, USA) were sorted into the following four fractions: i) p75NTR-positive cells in the G0-G1 phase (p75NTRpositive/G0-1); ii) p75NTR-positive cells in the S-G2-M phase (p75NTR-positive/S-G2-M); iii) p75NTR-negative cells in the G0-G1 phase (p75NTR-negative/G0-1); iv) p75NTR-negative cells in the S-G2-M phase (p75NTR-negative/S-G2-M). Each population was evaluated as follows.

RNA extraction, cDNA synthesis and real-time PCR. Total RNA was extracted using NucleoSpin ${ }^{\circledR}$ RNA (Macherey-Nagel GmbH \& Co.KG., Düren, Germany), according to the manufacturer's instructions. Quality and quantity of the total RNA were determined using NanoDrop ${ }^{\mathrm{TM}} 2000$ (Thermo Fisher Scientific, Wilmington, DE, USA) according to the manufacturer's instructions. cDNA was synthesized using the PrimeScript $^{\mathrm{TM}}$ II First Strand cDNA Synthesis kit (Takara Kyoto, Japan), according to the manufacturer's instructions. cDNA samples were amplified using Mx3000P real-time qPCR system (Agilent Technologies, Palo Alto, CA, USA) and
SYBR $^{\circledR}$ Premix Ex Taq ${ }^{\mathrm{TM}}$ II (Takara), according to the manufacturer's instructions. PCR was performed using the following protocol: $95^{\circ} \mathrm{C}$ for $15 \mathrm{sec}$, followed by 40 cycles of $95^{\circ} \mathrm{C}$ for $5 \mathrm{sec}$ and $60^{\circ} \mathrm{C}$ for $30 \mathrm{sec}$. mRNA expression was evaluated using $\Delta \Delta \mathrm{Ct}$ method, with GAPDH as an internal normalization control. Primers used for real-time PCR are as follows: p75NTR forward primer, AAGAAAAGTGGGCCAGTGTG and p75NTR reverse primer, AACAGTCCTTTGCAGGGT TG; Nanog forward primer, ATGCCTCACACGGAGACTGT and Nanog reverse primer, AAGTGGGTTGTTTGCCTTTG; p63 forward primer, CAGACTTGCCAGATCATCC and p63 reverse primer, CAGCATTGTCAGTTTCTTAGC; BMI-1 forward primer, CCACCTGATGTGTGTGCTTTG and BMI-1 reverse primer, TTCAGTAGTGGTCTGGTCTTGT; ABCG2 forward primer, AGCAGGGACGAACAATCATC and ABCG2 reverse primer, TTCCTGAGGCCAATAAGGTG; ERCC1 forward primer, GCCTCCGCTACCACAACCT and ERCC1 reverse primer, TCTTCTCTTGATGCGGCGA; GAPDH forward primer, ACCACAGTCCATGCCATCAC and GAPDH reverse primer, TCCACCACCCTGTTGCTGTA.

Cell cycle analysis. Cell cycle was analyzed by performing flow cytometry with BD CycleTest ${ }^{\mathrm{TM}}$ Plus DNA reagent kit (Becton-Dickinson, San Jose, CA, USA) following the specific protocol provided by the supplier. Data were analyzed using FCS4 Express cytometry (Becton-Dickinson).

Colony formation assay. KYSE-30 or KYSE-140 cells were sorted into 1,000 cells by using BD FACSAria ${ }^{\mathrm{TM}}$ II. The cells were plated in $60-\mathrm{mm}$ tissue culture dishes (Thermo Fisher Scientific). After 14 days of culturing, colonies were stained with Diff-Quik (Sysmex International Reagents, Co., Ltd., Kobe, Japan) and the number of colonies with a diameter of $>3 \mathrm{~mm}$ was counted.

Cell fate assay by using a fluorescent cell-tracing dye. KYSE-30 cells were stained with $10 \mu \mathrm{M}$ CellTrace ${ }^{\mathrm{TM}}$ Violet dye (Life Technologies, Carlsbad, CA, USA), according to the manufacturer's instructions, and the stained cells were incubated for 7 days at $37^{\circ} \mathrm{C}$ in an atmosphere of $5 \% \mathrm{CO}_{2}$. The cells were fixed with $4 \%$ paraformaldehyde in PBS for 15 min, followed by incubation with PBS containing $1 \%$ FCS for $30 \mathrm{~min}$ at room temperature. The cells were then incubated with anti-p75NGF receptor antibody (ab52987; dilution, 1:1,000; Abcam, Cambridge, UK) for $60 \mathrm{~min}$ at room temperature. Next, the cells were washed three times with PBS and were incubated with fluorescein isothiocyanate (FITC)conjugated goat anti-rabbit IgG H\&L (ab6717; dilution, 1:1,000; Abcam) for $30 \mathrm{~min}$ at room temperature. Finally, the cells were analyzed using a fluorescent microscope (BZ-8000; Keyence, Osaka, Japan).

Sensitivity to anticancer drug. Each cell population was cultured at a density of $2 \times 10^{3}$ cells/well in a 96-well plate (Thermo Fisher Scientific) containing DMEM/Ham's F-12 medium supplemented with $5 \% \mathrm{FCS}$ at $37^{\circ} \mathrm{C}$ in a humidified atmosphere of $5 \% \mathrm{CO}_{2}$. The cells were cultured overnight until they reached confluence, after which they were treated with or without (control) different concentrations of cisplatin (CDDP; Wako Pure Chemicals). Cell viability after $72 \mathrm{~h}$ of treatment 
with Cell Proliferation kit I (Roche Diagnostics GmbH, Mannheim, Germany) was determined by performing 3-(4, 5-dimethylthiazol-2-yl)-2,5-diphenyl-tetrazolium bromide (MTT) assay. Absorbance was measured at $595 \mathrm{~nm}$ by using FilterMax F5 (Molecular Devices, Tokyo, Japan). All assays were performed according to the manufacturer's protocols.

Tumorigenicity assay in nude mice. All mouse studies were carried out under the approval of the Institutional Review Board of the University of Toyama (no. A-2013 MED-11). Six-week-old athymic nude mice (BALB/CAN. Cg-Foxnl ${ }^{\text {nu}} / \mathrm{CrCrlj}$; Charles River Laboratories Japan Inc., Yokohama, Japan) were used for performing tumorigenicity assay with KYSE-30 cells. Sorted cells (density, 100-1,000 cells) were subcutaneously injected into the bilateral lumber of mice. The mice were sacrificed after 8 weeks, and the sites of subcutaneous tumor development were counted and tumor weights were measured.

Patients and surgical specimens. We analyzed 95 tumor specimens from patients with ESCC who underwent a surgery at our hospital from 1986 to 2016. Median follow-up time was 24 months (range, 1-134 months). The study included 84 male and 11 female patients, and with a median age of 67 years (range, 43-86 years). TNM stages (ver. 6) of the patients are as follows: stage I, 8 patients; stage IIA, 22 patients; stage IIB, 11 patients; stage III, 48 patients; and stage IV, 6 patients. In all, 23 patients received CDDP-based neoadjuvant chemotherapy, of which 19 patients received CDDP plus 5-fluorouracil (5-FU) therapy, and 4 patients received CDDP plus docetaxel and 5-FU therapy. The Institutional Review Board at the University of Toyama approved this study (no. 20-57).

Immunohistochemical analysis. Immunohistochemical staining was performed using anti-Ki-67 antibody (dilution, 1:100; Abcam) and human p75NTR monoclonal antibody against p75NGER (NGER 5; dilution, 1:100; Abcam). Immunostaining was performed using Envision Plus kits, horseradish peroxidase, or 3,3'-diaminobenzidine (Dako Cytomation, Kyoto, Japan), as recommended by the supplier. Counterstaining was performed using Mayer's hematoxylin.

The numbers of p75NTR-positive cells and all tumor cells with nuclear staining were counted in three random fields of each section. Then, the proportions of each cell type in each tumor were calculated. We classified tumors as positive when $>5 \%$ of the tumor cells were stained.

Double immunocytochemical staining of p75NTR and cell proliferation marker $\mathrm{Ki}-67$. Double immunocytochemical staining of p75NTR and Ki-67 was performed using BOND-III automated immunostainers (Leica Biosystems, Nussloch, Germany). Primary antibodies used for double immunocytochemical staining against p75NTR and Ki-67 were the same as used for immunohistochemical analysis.

Statistical analysis. Statistical analysis was performed using JMP 11.2.0 software (SAS Institute, Inc., Cary, NC, USA). Chi-square test and Fisher's exact test were used for performing statistical analyses, with $\mathrm{P}$-values of $<0.05$ being considered statistically significant. Kaplan-Meier method was used for performing survival analysis.

\section{Results}

Differentiation of cell subsets according to p75NTR expression and cell cycle status. Double immunocytochemical staining of p75NTR (red) and cell proliferation marker Ki-67 (brown), which are expressed in cells in the G1, S, G2 and $M$ phases of the cell cycle, differentiated KYSE-30 and KYSE-140 cells into p75NTR-positive/Ki-67-negative (white arrowhead), p75NTR-positive/Ki-67-positive (red arrowhead), p75NTR-negative/Ki-67-negative (blue arrowhead) and p75NTR-negative/Ki-67-positive (green arrowhead) cells (Fig. 1A). Precise cell counting showed that 11.4 and $15.7 \%$ KYSE-30 and KYSE-140 cells, respectively, showed p75NTR positivity and Ki-67-negativity (G0 phase). Furthermore, 21.8 and $33.8 \%$ p75NTR-positive and p75NTR-negative KYSE-30 cells, respectively, yielded negative results for Ki-67. Similarly, 36.5 and $43.1 \%$ p75NTR-positive and p75NTR-negative KYSE-140 cells, respectively, yielded negative results for Ki-67 (Fig. 1A).

Flow cytometric analysis of p75NTR expression showed positive staining in 26.8 and $36.4 \%$ KYSE-30 and KYSE-140, respectively (Fig. 1A). Flow cytometric analysis of cell cycle based on DNA content showed that 63.0 and 79.4\% p75NTRpositive and p75NTR-negative KYSE-30 cells, respectively, were in the G0/G1 phase and that 65.2 and $82.4 \%$ of p75NTRpositive and p75NTR-negative KYSE-140 cells, respectively, were in the G0/G1 phase (Fig. 1A). Thus, 16.9 and $23.7 \%$ p75NTR-positive KYSE-30 and KYSE-140 cells, respectively, were in the G0-G1 phase of the cell cycle.

Flow cytometric analysis identified four distinct cell subsets, i.e., p75NTR-positive/G0-1, p75NTR-positive/SG2-M,p75NTR-negative/G0-1 and p75NTR-negative/ S-G2-M cells, based on p75NTR expression and cell cycle status.

Flow cytometric cell sorting based on p75NTR expression and cell cycle status. To isolate viable p75NTR-positive/ G0-1, p75NTR-positive/S-G2-M, p75NTR-negative/G0-1, and p75NTR-negative/S-G2-M cells, KYSE-30 and KYSE-140 cells were fractionated into p75NTR-positive and p75NTRnegative cells by using the APC-conjugated anti-p75NTR antibody, followed by fractionation into cells in the G0-G1 and S-G2-M phases by using a fluorescent DNA-staining dye DCV (Fig. 1B).

Expression of p75NTR in the four cell subsets sorted by flow cytometry was confirmed by performing real-time PCR. The mRNA expression of p75NTR was significantly higher in p75NTR-positive/G0-1 and p75NTR-positive/S-G2-M cells than in p75NTR-negative/G0-1 or p75NTR-negative/S-G2-M cells (Fig. 1C).

Cell cycle status of each cell subset was confirmed using another DNA-staining dye (BD CycleTest ${ }^{\mathrm{TM}}$ Plus DNA reagent kit). Results showed that 93.29 and $92.88 \%$ in p75NTR-positive and p75NTR-negative KYSE-30 cells, respectively, were in the G0-G1 phase and 92.74 and $94.29 \%$ p75NTR-positive and p75NTR-negative KYSE-140 cells, respectively, were in the G0-G1 phase (Fig. 1C). Furthermore, 99.9 and $93.43 \%$ p75NTR-positive and p75NTR-negative KYSE-30 cells, respectively, were in the S-G2-M phase, and 99.9 and $94.43 \%$ p75NTR-positive and p75NTR-negative KYSE-140 cells, respectively, were in the S-G2-M phase (Fig. 1C). 

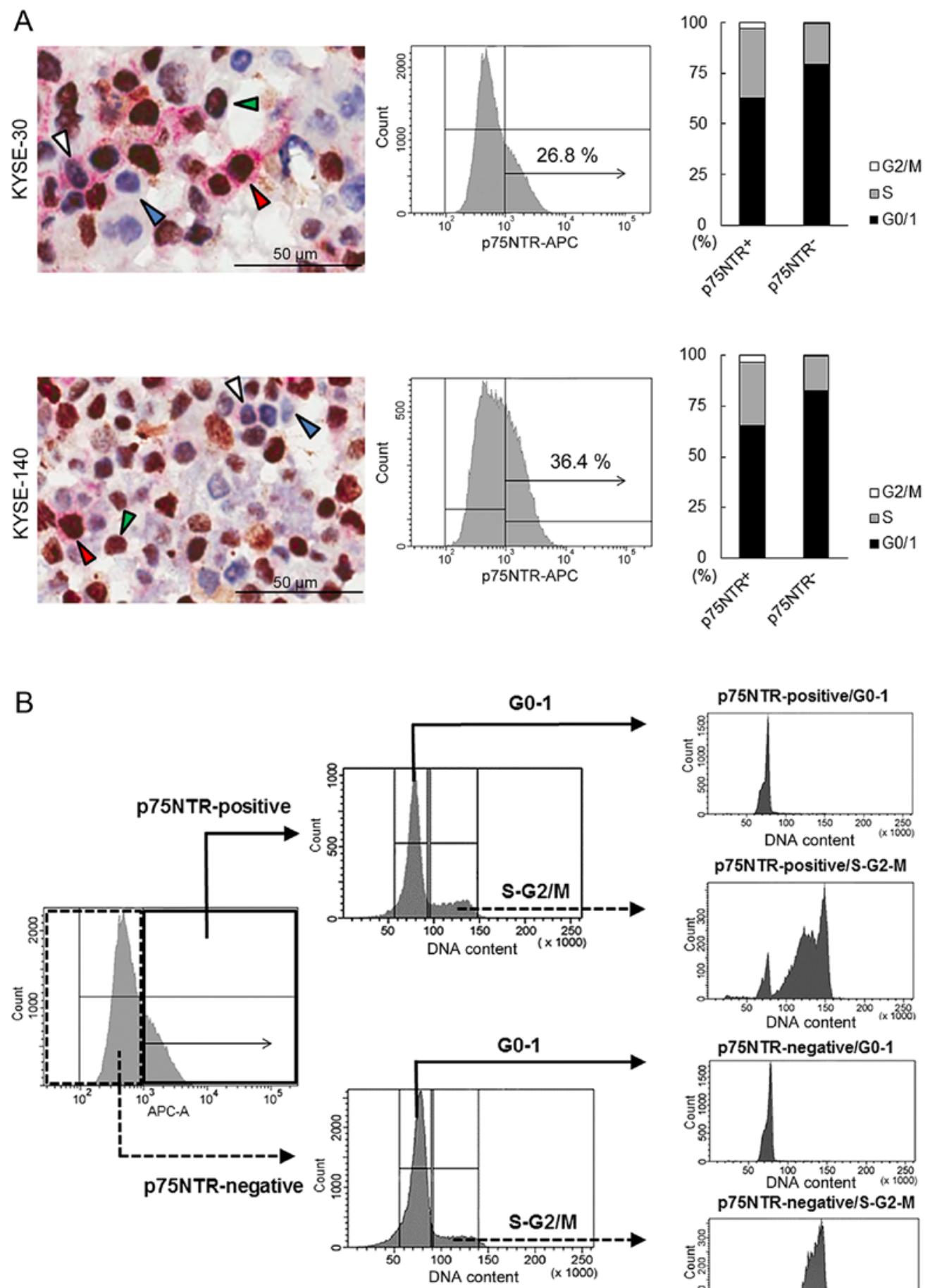

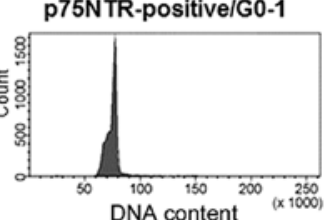

p75NTR-positive/S-G2-M

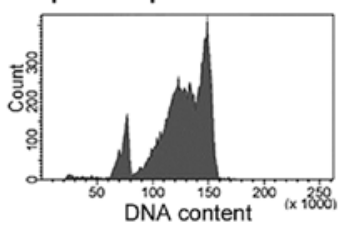

p75NTR-negative/G0-1

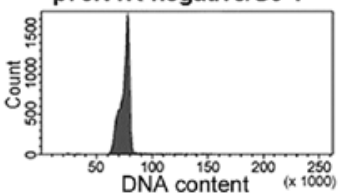

p75NTR-negative/S-G2-M

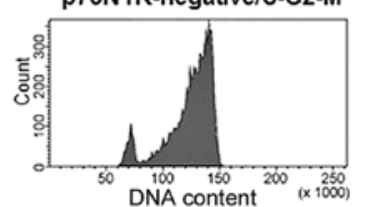

Figure 1. Flow cytometric cell sorting based on p75NTR expression and cell cycle status. (A) The left panels show representative results of double immunocytochemical staining of Ki-67 (brown) and p75NTR (red) in KYSE-30 (upper panel) and KYSE-140 cells (lower panel). Nuclei are stained with hematoxylin. The white, red, blue and green arrowheads indicate p75NTR-positive/Ki-67-negative, p75NTR-positive/Ki-67-positive, p75NTR-negative/Ki-67-negative and p75NTR-negative/Ki-67-positive cells, respectively. The middle panels show representative results of flow cytometric analysis of p75NTR expression in KYSE-30 (upper panel) and KYSE-140 cells (lower panel). Cut-off levels were set based on control IgG, and percentages of positive cells are shown in the panels. The right panels show cell cycle distribution in p75NTR-positive and p75NTR-negative KYSE-30 (upper panel) and KYSE-140 cells (lower panel). Live cells were analyzed using a DNA-staining dye (DCV) and FACSAria II. The proportion of cells in each phase of the cell cycle is shown. (B) Diagram showing the steps of flow cytometric cell fractionation based on p75NTR expression and cell cycle status. KYSE-30 and KYSE-140 cells were fractionated into p75NTR-positive and p75NTR-negative cells. Next, each cell subset was fractionated into cells in the G0-G1 and S-G2-M phases of the cell cycle by using a fluorescent DNA-staining dye (DCV).

Stem cell phenotype of fractionated cell subsets. Results of real-time PCR showed that expression of stem cell-related genes such as Nanog, BMI-1 and p63 was significantly higher in p75NTR-positive/G0-1 KYSE-30 and KYSE-140 cells than 

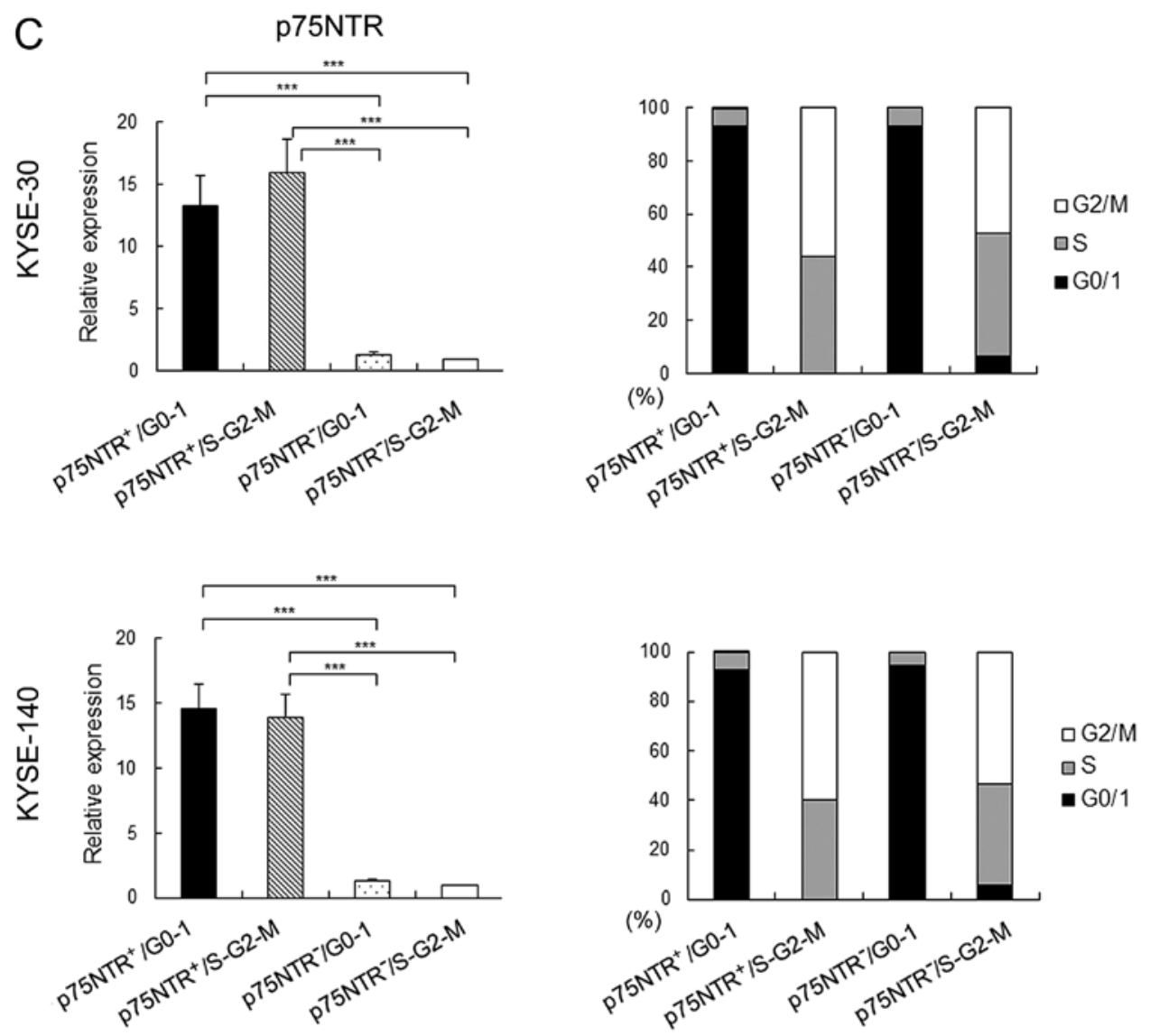

Figure 1. Continued. (C) The left panels show p75NTR expression in the four cell subsets sorted from KYSE-30 (upper panel) and KYSE-140 cells (lower panel), as determined by performing real-time PCR. The right panels show cell cycle status of each of the four cell subsets sorted from KYSE-30 (upper panel) and KYSE-140 cells (lower panel), as determined using another fluorescent DNA-staining dye (BD CycleTest ${ }^{\mathrm{TM}}$ Plus DNA reagent kit). The error bars represent standard error of mean; ${ }^{* * *} \mathrm{P}<0.001$.

Table I. In vivo tumor development after injecting p75NTRpositive/G0-1, p75NTR-positive/S-G2-M, p75NTR-negative/ G0-1 and p75NTR-negative/S-G2-M KYSE-30 cells into nude mice.

\begin{tabular}{lccc}
\hline & \multicolumn{3}{c}{ Tumor incidence } \\
\cline { 2 - 4 } No. of cells injected & 1,000 & 300 & 100 \\
\hline KYSE-30 & & & \\
p75NTR-positive/G0-1 & $6 / 6$ & $4 / 6$ & $5 / 6$ \\
p75NTR-positive/S-G2-M & $4 / 6$ & $4 / 6$ & $2 / 6$ \\
p75NTR-negative/G0-1 & $3 / 6$ & $2 / 6$ & $0 / 6$ \\
p75NTR-negative/S-G2-M & $2 / 6$ & $1 / 6$ & $0 / 6$ \\
\hline
\end{tabular}

in the other cell subsets, including p75NTR-positive/S-G2-M fraction KYSE-30 and KYSE-140 cells (Fig. 2A). Furthermore, p75NTR-positive/G0-1 KYSE-30 and KYSE-140 cells showed the highest colony formation ability, with statistical significance, among the four cell subsets, while p75NTRpositive/S-G2-M KYSE-30 and KYSE-140 cells showed the second highest colony formation ability (Fig. 2B).

To detect slow-cycling p75NTR-positive cells, KYSE-30 cells were stained with a fluorescent cell-tracing reagent, which diffuses into cells and covalently binds to intracellular amines. After labeling, all the adherent KYSE-30 cells showed fluorescent staining on culture day 1 (Fig. 2C). However, the number of unstained cells increased by culture day 7 because the concentration of the label decreased after cell division. However, a small number of p75NTR-positive cells retained the label, suggesting that these cells divided less frequently (Fig. 2C). Cell counting showed that $0.9 \%$ of all the cells retained the label, and $3.4 \%$ of p75NTR-positive cells were label-retaining cells. In contrast, no label-retention was observed in p75NTR-negative cells (Fig. 2C).

Drug resistance ability of the fractionated cell subsets. Results of real-time PCR showed that the expression of ATP-binding cassette sub-family G member 2 (ABCG2) was significantly higher in p75NTR-positive cells than in p75NTR-negative cells, irrespective of their cell cycle status (Fig. 3A). On the other hand, expression of excision repair cross-complementation group 1 (ERCC1), which contributes to resistance against platinum-based chemotherapeutic drugs, was significantly higher in p75NTR-positive/G0-1 cells than in other cell subsets, including p75NTR-positive/SG2-M cells (Fig. 3A). Results of the MTT assay showed that the viability of total cells decrease by CDDP treatment dose-dependently both in KYSE-30 and KYSE-40. However, the viability of p75NTR-positive/G0-1 KYSE-30 cells was 
A
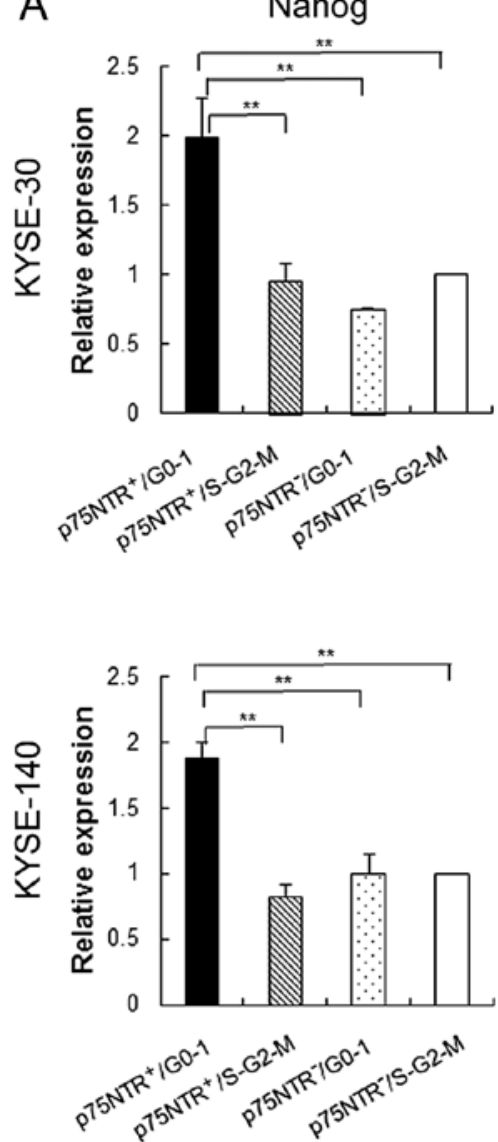
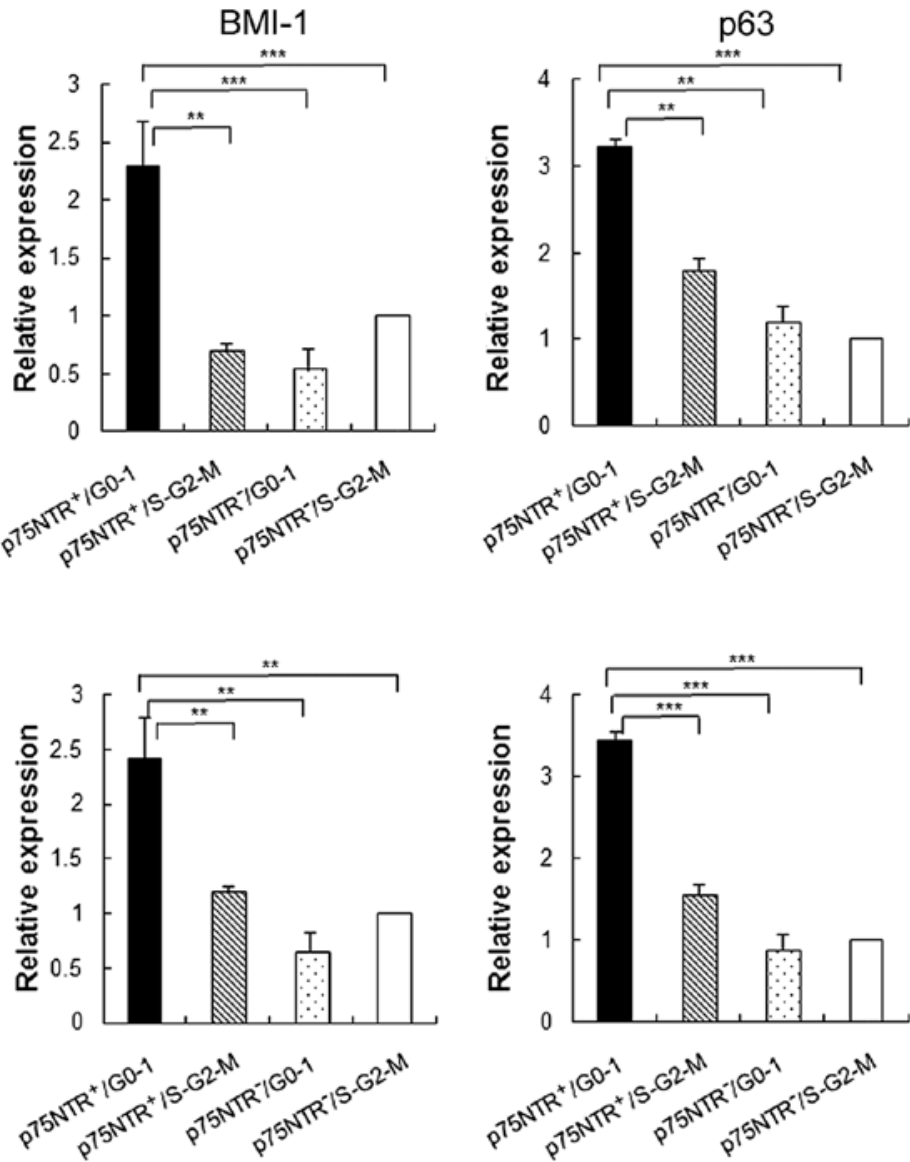

Figure 2. In vitro stem cell phenotypes of the fractionated cell subsets. (A) Expression of stem cell-related genes in the four cell subsets sorted from KYSE-30 (upper panel) and KYSE-140 cells (lower panel) was determined by performing real-time PCR. Expression levels of these genes are shown relative to their expression levels in p75NTR-negative/S-G2-M cells. The error bars represent standard error of mean; ${ }^{* *} \mathrm{P}<0.01,{ }^{* * *} \mathrm{P}<0.001$.

significantly higher than that of the other cell subsets at all CDDP concentrations. Furthermore, the viability of p75NTRpositive/G0-1 KYSE-140 cells was significantly higher than that of the other cell subsets at CDDP concentrations of 100 and $200 \mu \mathrm{M}$ (Fig. 3B).

Tumorigenicity in nude mice. Subcutaneous injection of as few as 100 p75NTR-positive/G0-1 KYSE-30 cells into nude mice resulted in tumor development at 5 of $6(83.3 \%)$ injection sites after 8 weeks, while that of p75NTR-positive/S-G2-M KYSE-30 cells resulted in tumor development at 2 of $6(33.3 \%)$ injection sites. Injection of 100 p75NTR-negative/G0-1 or p75NTR-negative/S-G2-M cells did not result in tumor development (Table I). The weights of tumors derived from p75NTR-positive/G0-1 cells were higher than those of tumors derived from p75NTR-positive/S-G2-M cells (Fig. 4A).

Histological examination of xenograft tumors showed that a large percentage $(83.4 \%)$ of cells in tumors derived from 300 p75NTR-positive/G0-1 cells showed p75NTR (red) and Ki-67 (brown) positivity. The proportion of other cell fractions was as follows: p75NTR-positive/Ki-67-negative cells, 9.3\%; p75NTR-negative/Ki-67-positive cells, 3.5\%; and p75NTRnegative/Ki-67-negative cells, 3.8\% (Fig. 4B).

Identification of cell subsets based on p75NTR expression and cell cycle status in surgically resected ESCC specimens and their clinicopathological significance. The expression of p75NTR was positive ( $>5 \%$ of the cancer cells were stained in tumor) in 39 of 95 (41.1\%) ESCC specimens, with positive staining observed in the first few layers of infiltrative margin (Fig. 5A). Correlation between p75NTR expression and various prognostic factors is summarized in Table II. p75NTR was correlated with tumor depth $(\mathrm{P}=0.032)$ and neoadjuvant chemotherapy $(\mathrm{P}=0.031)$. However, no significant correlation was observed between p75NTR expression and other factors such as age, sex, tumor location, lymph node metastasis, distant metastasis, TNM classification, histology and postoperative tumor recurrence. Kaplan-Meier survival curves showed that p75NTR expression was correlated with a favorable overall survival (Fig. 5B).

Double immunohistochemical staining of p75NTR and Ki-67 was performed in 20 of 39 p75NTR-positive patients who provided appropriate additional specimens. Representative images of well differentiated and poorly differentiated tumors are shown in Fig. 5C. p75NTR-positive staining was apparent in the first one to two layers from the infiltrative margin in well differentiated tumors. In contrast, p75NTR-positive staining was diffusely distributed in poorly differentiated tumors. Double immunohistochemical staining of p75NTR (red) and Ki-67 (brown) helped in distinguishing p75NTR-positive/ Ki-67-negative (white arrowhead), p75NTR-positive/Ki-67positive (red arrowhead), p75NTR-negative/Ki-67-negative 

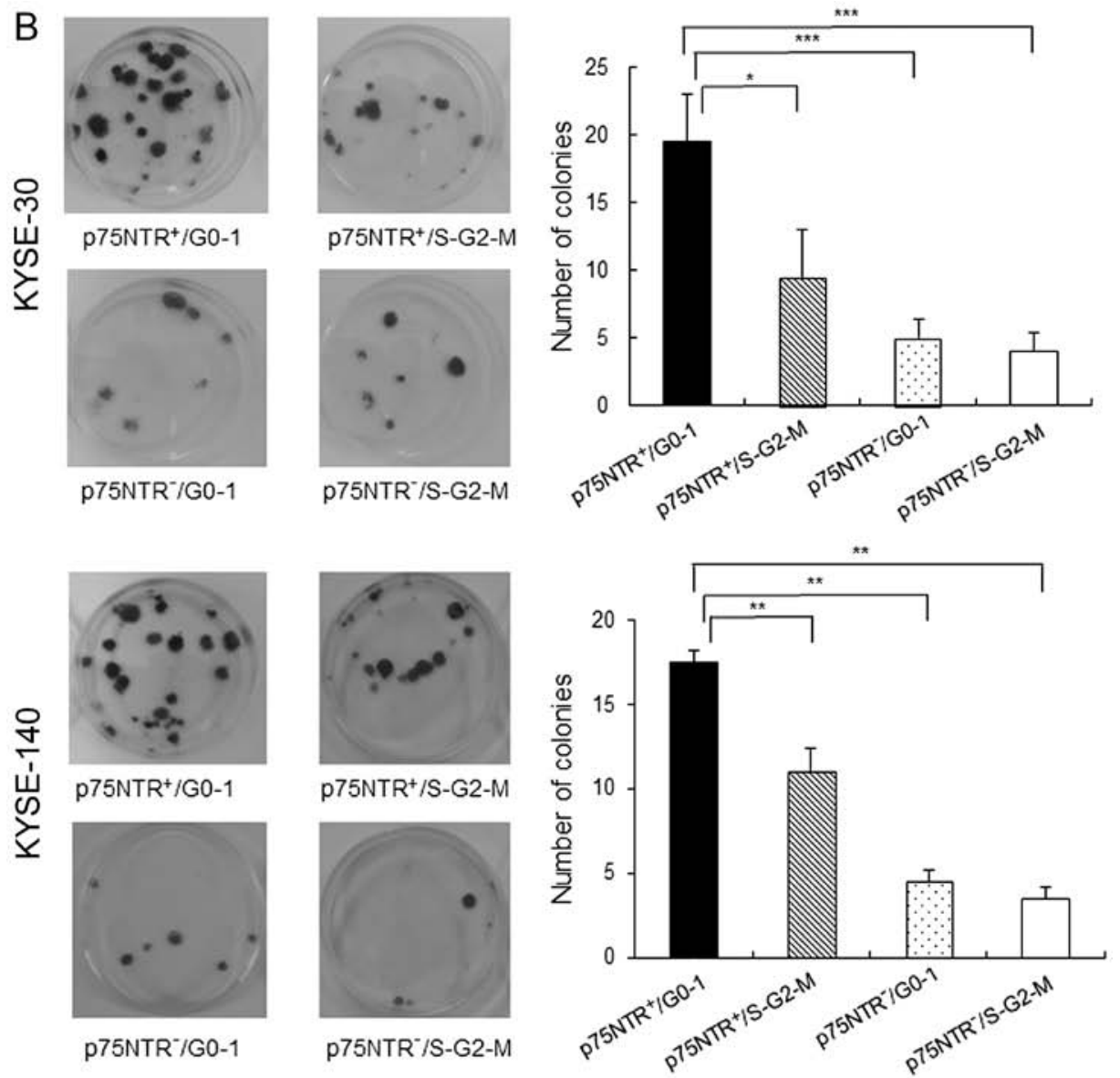

p75NTR $/$ G0-1
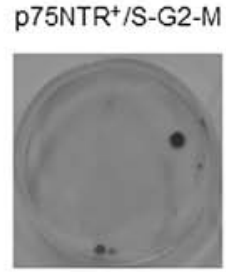

p75NTR//S-G2-M

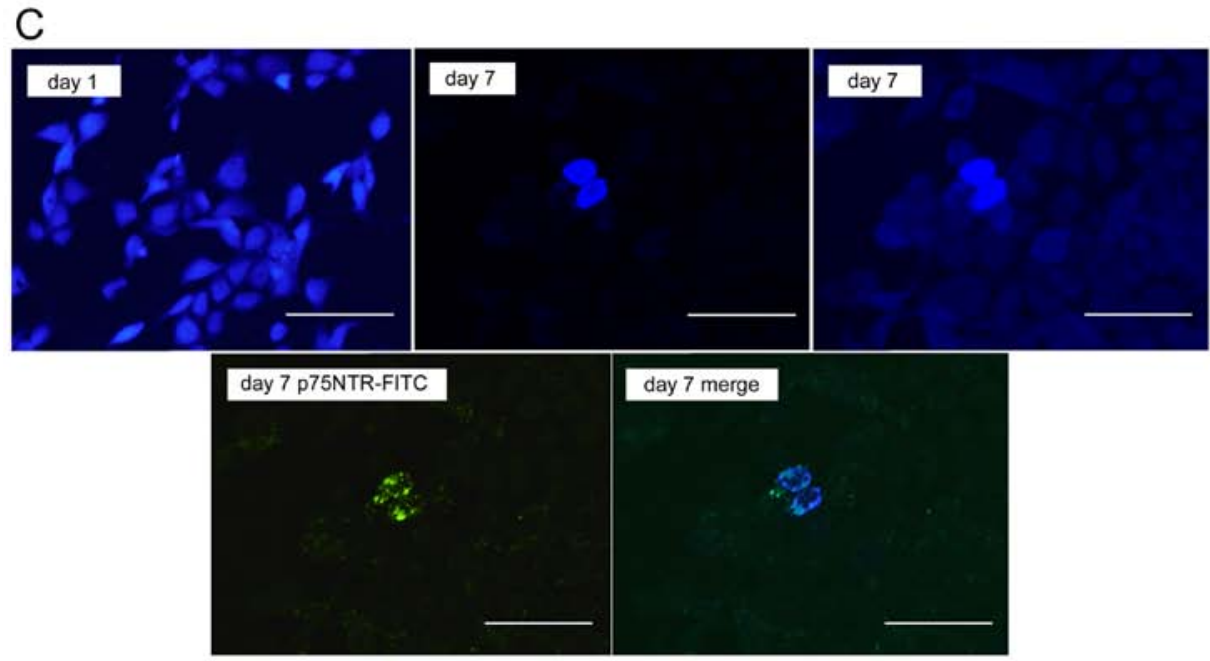

Figure 2. Continued. (B) Colony formation ability of the four cell subsets sorted from KYSE-30 (upper panels) and KYSE-140 cells (lower panels). Crystal violet staining of the colonies formed at 2 weeks after seeding (left panels). Mean number of colonies with a diameter of $>3 \mathrm{~mm}$ was counted (right panels). The error bars represent standard error of mean; ${ }^{*} \mathrm{P}<0.05,{ }^{* *} \mathrm{P}<0.01,{ }^{* * * *} \mathrm{P}<0.001$. (C) Expression of p75NTR in label-retaining KYSE-30 cells, as determined using the fluorescent cell-tracing reagent. Fluorescently labeled cells (violet) were detected using a fluorescence microscope on days 1 (upper left panel) and 7 (upper mid panel: original photo, upper right panel: the contrast was decreased to visualize cells with decreased label-retention). On day 7, cell culture plate was stained with FITC-conjugated anti-p75NTR antibody and positive cells (green) were detected using fluorescence microscope (lower left panel). The merged image is shown in the lower right panel; scale bar, $50 \mu \mathrm{m}$.

(blue arrowhead) and p75NTR-negative/Ki-67-positive (green arrowhead) cells. The number of cells belonging to each subset was counted for each section (Fig. 5C). The median proportion of p75NTR-positive cells in 20 tumors was $28.7 \%$ (range, 12.9-94.0\%) and that of p75NTR-positive/Ki-67-negative cells in 20 tumors was $13.2 \%$ (range, $3.0-80.1 \%$ ).
The relationship between the proportion of p75NTR-positive/Ki-67-negative cells in tumors and clinicopathological factors is summarized in Table III. High proportion of p75NTRpositive/Ki-67-negative cells was correlated with poorly differentiated histology $(\mathrm{P}=0.02)$, postoperative tumor recurrence $(\mathrm{P}=0.007)$ and with the proportion of $\mathrm{p} 75 \mathrm{NTR}$-positive 
Table II. Relationship between p75NTR expression and clinicopathological characteristics of patients with ESCC.

\begin{tabular}{|c|c|c|c|}
\hline & \multicolumn{3}{|c|}{ p75NTR } \\
\hline & $\begin{array}{c}\text { Positive } \\
(\mathrm{n}=39)\end{array}$ & $\begin{array}{c}\text { Negative } \\
(\mathrm{n}=56)\end{array}$ & P-value \\
\hline \multicolumn{4}{|l|}{ Sex } \\
\hline Male & 34 & 50 & 0.756 \\
\hline Female & 5 & 6 & \\
\hline \multicolumn{4}{|l|}{ Age (years) } \\
\hline$\geq 65$ & 23 & 31 & 0.834 \\
\hline$<65$ & 16 & 25 & \\
\hline \multicolumn{4}{|l|}{ Site } \\
\hline $\mathrm{Ce}-\mathrm{Ut}$ & 4 & 6 & 0.943 \\
\hline Mt-Ae & 35 & 50 & \\
\hline \multicolumn{4}{|l|}{ pT } \\
\hline $\mathrm{T} 1-\mathrm{T} 2$ & 19 & 15 & $0.032^{\mathrm{a}}$ \\
\hline T3-T4 & 20 & 41 & \\
\hline \multicolumn{4}{|l|}{$\mathrm{pN}$} \\
\hline N0 & 13 & 22 & 0.667 \\
\hline N1-3 & 26 & 34 & \\
\hline \multicolumn{4}{|l|}{ M } \\
\hline M0 & 37 & 52 & 0.691 \\
\hline M1 & 2 & 4 & \\
\hline \multicolumn{4}{|l|}{ pStage } \\
\hline I-II & 20 & 21 & 0.211 \\
\hline III-IV & 19 & 35 & \\
\hline \multicolumn{4}{|l|}{ Histology } \\
\hline Well-mod & 30 & 46 & 0.601 \\
\hline Poor & 9 & 10 & \\
\hline \multicolumn{4}{|c|}{ Neoadjuvant chemotherapy } \\
\hline Yes & 14 & 9 & $0.031^{\mathrm{a}}$ \\
\hline No & 25 & 47 & \\
\hline \multicolumn{4}{|c|}{ Postoperative tumor recurrence } \\
\hline Yes & 7 & 17 & 0.231 \\
\hline No & 32 & 39 & \\
\hline
\end{tabular}

${ }^{\mathrm{a}} \mathrm{P}<0.05$.

cells in tumors $(\mathrm{P}=0.01)$. Kaplan-Meier survival curves showed a trend of unfavorable postoperative prognosis in patients who had tumor with higher proportion of p75NTR-positive/Ki-67negative cells, although there was no statistically significant correlation (Fig. 5D).

\section{Discussion}

Recent studies involving ESCC cell lines have shown that p75NTR is expressed in a cell subset possessing CSC properties such as colony formation ability, tumorigenicity in mouse
Table III. Relationship between p75NTR and Ki-67 expression and clinicopathological characteristics of patients with ESCC.

\begin{tabular}{|c|c|c|c|}
\hline & \multicolumn{3}{|c|}{$\begin{array}{l}\text { Proportion of p75NTR-positive } \\
\text { Ki-67-negative cells }\end{array}$} \\
\hline & $\begin{array}{c}\text { Low } \\
(<13.2 \%) \\
(\mathrm{n}=10)\end{array}$ & $\begin{array}{c}\text { High } \\
(\geq 13.2 \%) \\
(\mathrm{n}=10)\end{array}$ & P-value \\
\hline \multicolumn{4}{|l|}{ Sex } \\
\hline Male & 8 & 9 & 0.528 \\
\hline Female & 2 & 1 & \\
\hline \multicolumn{4}{|l|}{ Age (years) } \\
\hline$\geq 65$ & 4 & 5 & 0.653 \\
\hline$<65$ & 6 & 5 & \\
\hline \multicolumn{4}{|l|}{ Site } \\
\hline $\mathrm{Ce}-\mathrm{Ut}$ & 6 & 5 & 0.653 \\
\hline Mt-Ae & 4 & 5 & \\
\hline \multicolumn{4}{|l|}{ pT } \\
\hline $\mathrm{T} 1-\mathrm{T} 2$ & 6 & 4 & 0.371 \\
\hline T3-T4 & 4 & 6 & \\
\hline \multicolumn{4}{|l|}{$\mathrm{pN}$} \\
\hline No & 3 & 1 & 0.255 \\
\hline N1-3 & 7 & 9 & \\
\hline \multicolumn{4}{|l|}{ M } \\
\hline M0 & 10 & 10 & N/A \\
\hline M1 & 0 & 0 & \\
\hline \multicolumn{4}{|l|}{ pStage } \\
\hline I-II & 3 & 3 & 1.00 \\
\hline III-IV & 7 & 7 & \\
\hline \multicolumn{4}{|l|}{ Histology } \\
\hline Well-mod & 8 & 3 & $0.021^{\mathrm{a}}$ \\
\hline Poor & 2 & 7 & \\
\hline \multicolumn{4}{|c|}{ Neoadjuvant chemotherapy } \\
\hline Yes & 5 & 6 & 0.653 \\
\hline No & 5 & 4 & \\
\hline \multicolumn{4}{|c|}{$\begin{array}{l}\text { Postoperative tumor } \\
\text { recurrence }\end{array}$} \\
\hline Yes & 1 & 7 & $0.007^{\mathrm{b}}$ \\
\hline No & 9 & 3 & \\
\hline \multicolumn{4}{|c|}{ p75NTR-positive cells (\%) } \\
\hline Low $(<29.1 \%)$ & 8 & 2 & $0.006^{\mathrm{b}}$ \\
\hline $\operatorname{High}(\geq 29.1 \%)$ & 2 & 8 & \\
\hline
\end{tabular}

$\mathrm{N} / \mathrm{A}$, not applicable. ${ }^{\mathrm{a}} \mathrm{P}<0.05 ;{ }^{\mathrm{b}} \mathrm{P}<0.01$.

xenograft models and chemoresistance (15-18). However, p75NTR-positive cells are heterogeneous in terms of cell surface marker expression and cell cycle status (18). Double 
A
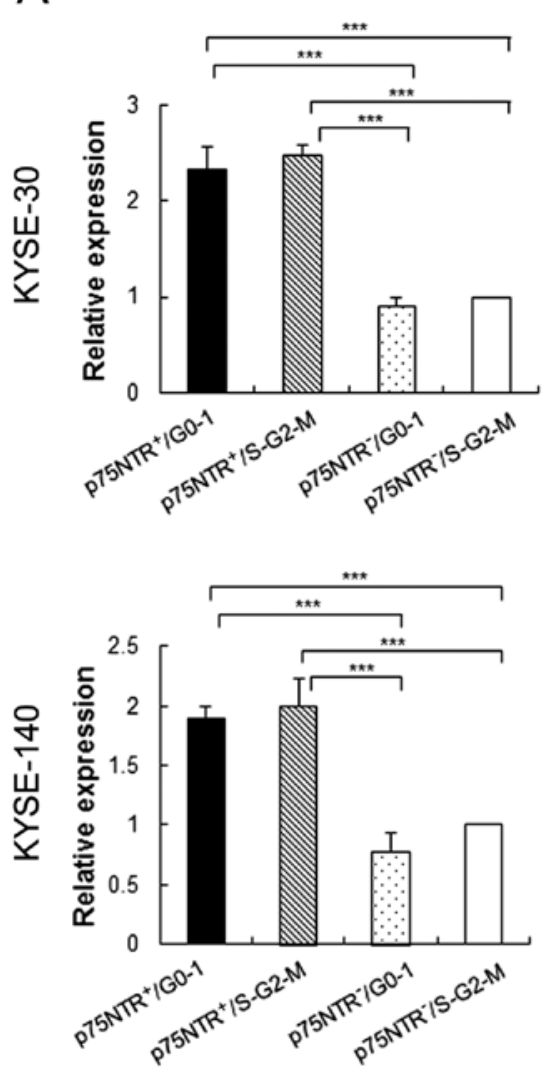
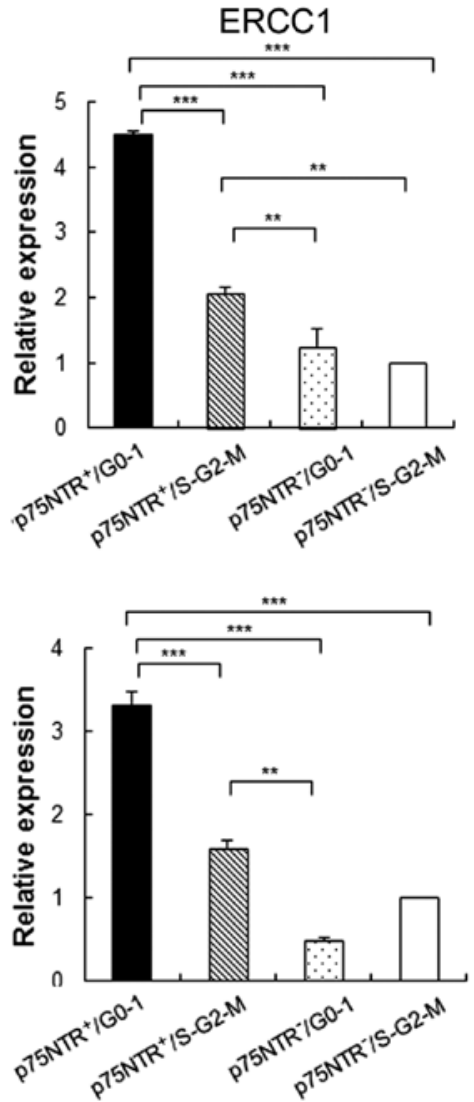

B
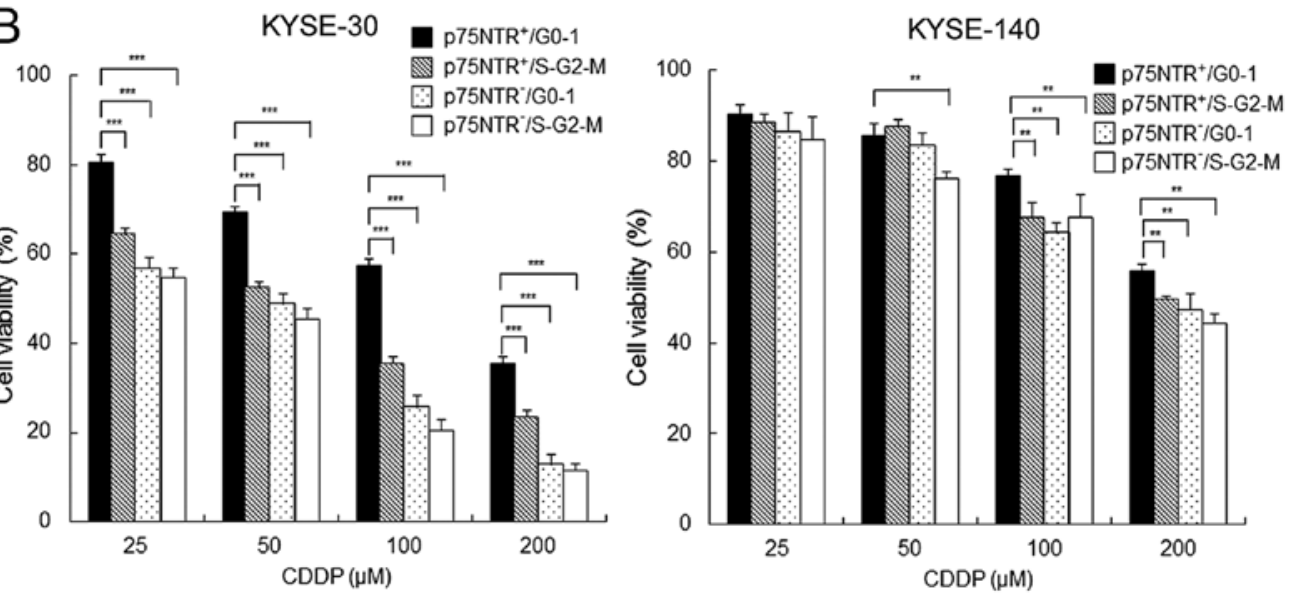

Figure 3. Drug resistance of the fractionated cell subsets. (A) Expression of drug resistance-related genes in the four cell subsets sorted from KYSE-30 (upper panels) and KYSE-140 cells (lower panels) was determined by performing real-time PCR. Expression levels of these genes are shown relative to their expression levels in $\mathrm{p} 75 \mathrm{NTR}-$ negative/S-G2-M cells. The error bars represent standard error of mean; ${ }^{* * *} \mathrm{P}<0.01,{ }^{* * *} \mathrm{P}<0.001$. (B) Fractionated KYSE-30 (left panel) and KYSE-140 (right panel) cells in 96 well plates $\left(2 \times 10^{3}\right.$ cells/well) were treated with CDDP $(25,50,100$ or $200 \mu \mathrm{M})$ for 3 days, and their viability was determined by performing the MTT assay. The error bars represent standard error of mean; ${ }^{* *} \mathrm{P}<0.01,{ }^{* * * *} \mathrm{P}<0.001$.

immunostaining performed in the present study detected four distinct cell subsets based on the expression of p75NTR and Ki-67 and showed that 11.4 and 15.7\% KYSE-30 and KYSE140 cells, respectively, were p75NTR-positive quiescent cells (which are present in the resting phase of the cell cycle). Flow cytometric cell sorting based on p75NTR expression and by using a fluorescent DNA-staining dye showed that p75NTRpositive/G0-1 cells, but not p75NTR-positive/S-G2-M cells, showed strong expression of stem-related genes (Nanog, BMI-1 and p63), high colony formation ability, high tumorigenicity in a mouse xenograft model, and strong chemoresistance, indicating that $\mathrm{p} 75 \mathrm{NTR}$-positive/G0-1 cells represented a more enriched CSC population in ESCC. The above results along with the recent studies that detected quiescent CSCs with enhanced CSC phenotypes in solid tumors (9-11), suggest that p75NTR-positive/G0-1 cells are quiescent CSCs in ESCC.

Results of Ki-67 immunocytochemical analysis and flow cytometric cell cycle analysis did not show a significant difference between p75NTR-positive and p75NTR-negative cells with respect to cell cycle distribution. This is consistent 

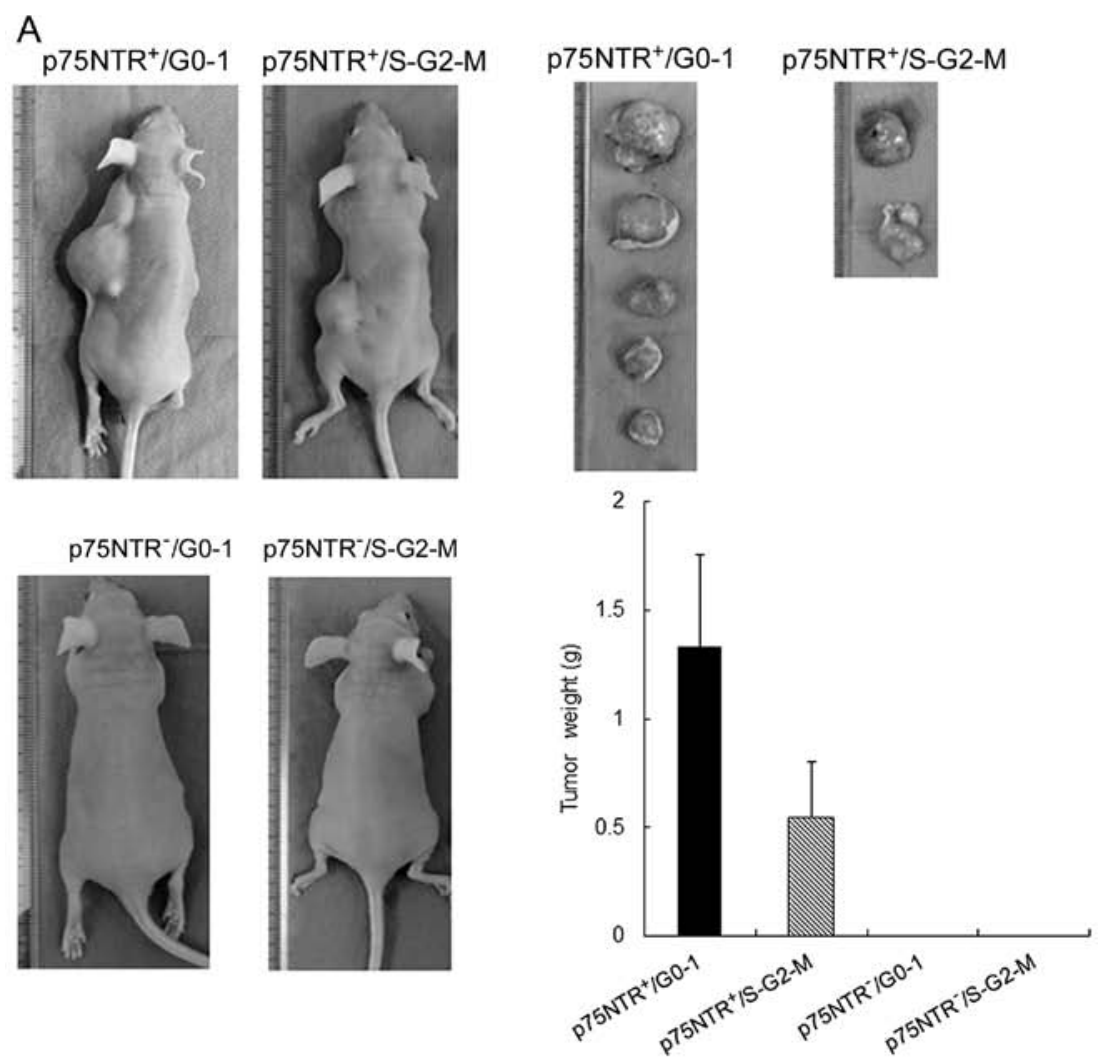

B
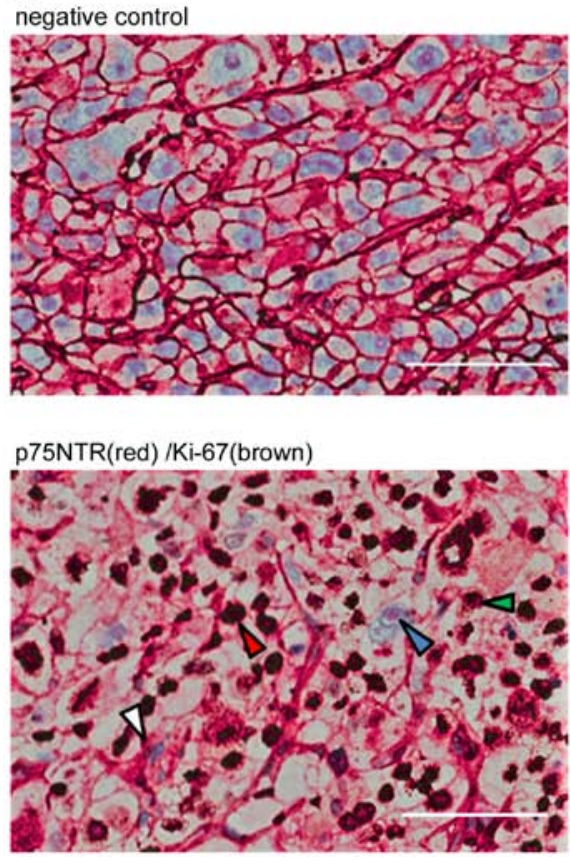

p75NTR $/$ G0-1

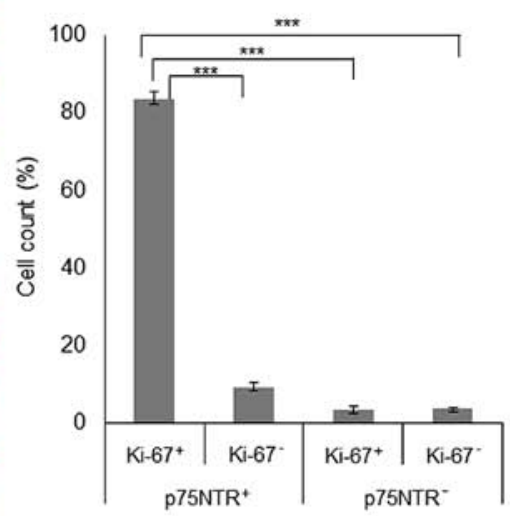

Figure 4. In vivo tumorigenicity analysis of the fractionated cell subsets. (A) Representative images of nude mice with subcutaneous tumors derived from 100 fractionated KYSE-30 cell subsets and tumors derived from these cell subsets (left panels). Mean tumor weight at each site (right panels). The error bars represent standard error of mean. (B) Representative results of double immunohistochemical staining of Ki-67 and p75NTR in subcutaneous tumors derived from 300 fractionated KYSE-30 cell subsets. Negative control (left upper panel). The white, red, blue and green arrowheads indicate p75NTR-positive/Ki-67negative, p75NTR-positive/Ki-67-positive, p75NTR-negative/Ki-67-negative and p75NTR-negative/Ki-67-positive cells, respectively (left lower panel); scale bar, $50 \mu \mathrm{m}$. The number of cells in each subset was counted (right panel). The error bars represent standard error of mean; *** $\mathrm{P}<0.001$.

with the results of previous studies on ESCC (18) and pancreatic cancer cell lines (22), indicating that cell cycle status alone cannot be used to isolate quiescent CSCs. In the present study, cell sorting along with the detection of cell cycle status and proposed CSC marker p75NTR enabled us to differentiate between quiescent CSCs and quiescent non-CSCs. A previous study reported that squamous differentiation marker involucrin is highly expressed in a subpopulation 

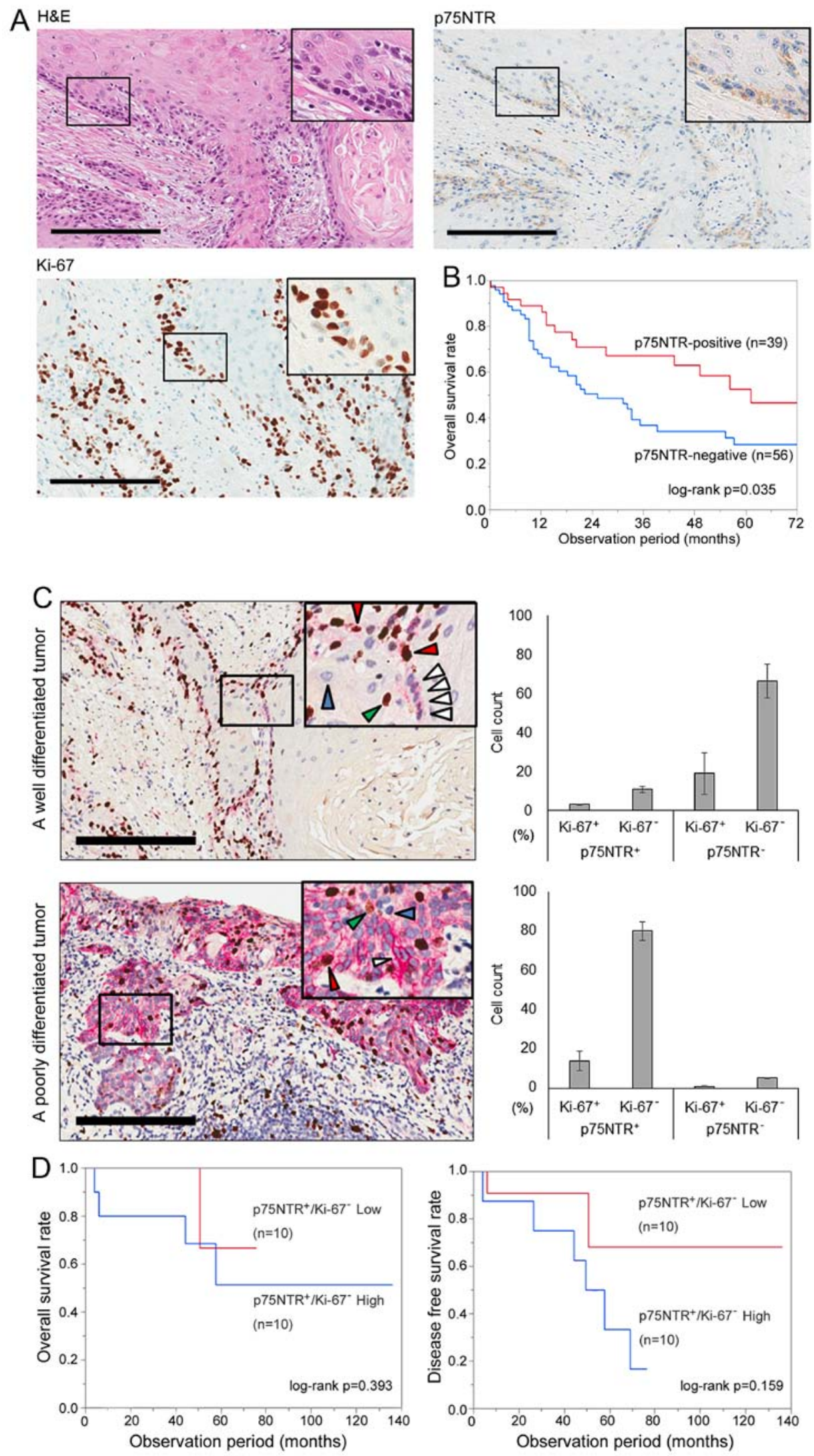

Figure 5. Identification of cell subsets based on p75NTR expression and cell cycle status in surgically resected ESCC specimens. (A) Representative results of positive staining for H\&E, p75NTR and Ki-67 in a serial section of a well differentiated ESCC specimen. Original magnification, x200; scale bar, $200 \mu \mathrm{m}$. (B) Correlation between p75NTR expression and overall survival after surgery. In all, 95 patients with ESCC were analyzed by performing Kaplan-Meier survival analysis (log-rank test). (C) Representative results of double immunohistochemical staining of p75NTR (red) and Ki-67 (brown) in a well differentiated tumor specimen (upper left panel) and a poorly differentiated tumor specimen (lower left panel). Nuclei were stained with hematoxylin. The white, red, blue and green arrowheads indicate p75NTR-positive/Ki-67-negative, p75NTR-positive/Ki-67-positive, p75NTR-negative/Ki-67-negative and p75NTR-negative/ Ki-67-positive cells, respectively; scale bar, $200 \mu \mathrm{m}$. The number of cells of each subset was counted (right panels). The error bars represent standard error of mean. (D) Correlation between the proportion of p75NTR-positive/Ki-67-negative cells in tumors and overall survival after curative surgery. In all, 20 patients with p75NTR-positive ESCC tumors were analyzed by performing Kaplan-Meier survival analysis (log-rank test). 
of p75NTR-negative cells, most of which are in the G0/G1 phase of the cell cycle (18). p75NTR-negative/G0-1 cells were suggested to include cells that withdrew from the cell cycle as well as cells that showed squamous differentiation or cellular senescence.

In the present study, 21.8 and $36.5 \%$ p75NTR-positive KYSE-30 and KYSE-140 cells, respectively, showed Ki-67 negativity. Flow cytometric analysis showed that 63.0 and $65.2 \%$ p75NTR-positive KYSE-30 and KYSE-140 cells, respectively, were in the G0/G1 phase of the cell cycle. Ki-67 is a marker of cell proliferation and is expressed during all the active phases of the cell cycle (G1, S, G2 and M) but is not expressed in resting (G0) cells (23). Flow cytometric cell cycle assays based on the quantification of DNA content by using DNA-binding dye-detected cells in the G0/G1, S and G2/M phases (24). Flow cytometric cell cycle assay performed in the present study showed that $\sim 33.3 \%(21.8 / 63.0)$ and $56.0 \%$ (36.5/65.2) p75NTR-positive KYSE-30 and KYSE-140 cells, respectively, were in the G0 phase. A novel assay system that enables the isolation of viable CSCs in the G0 phase may allow more precise characterization of quiescent CSCs.

In previous studies, slow-cycling CSCs were detected by performing label-retention assays with thymidine analog BrdU, which incorporates into newly synthesized DNA, or with lipophilic labeling dyes such as DiI and PKH26, which incorporate into the cell membrane (9-11). After several weeks in culture, infrequently dividing slow-cycling cells that retain originally incorporated labels are detected as label-retaining cells; in contrast, label concentration decreases in fast-cycling bulk tumor cells after each round of cell division (25). In the present study, absence of Ki-67 immunoreactivity and detection of cells in the G0 phase indicate quiescence of cells just at the time of analysis but does not indicate a continuously slow-cycling phenotype, which depends on the doubling time of quiescent cells (i.e., the duration for which the cells stay in the resting phase of the cell cycle). However, results of label-retention assay performed in the present study by using a fluorescent cell-tracing reagent showed label-retention in $3.4 \%$ p75NTR-positive cells, but not in p75NTR-negative cells, suggesting relatively slow cycling of some p75NTR-positive/ G0-1 cells. We performed flow cytometric cell sorting based on p75NTR expression and by using DNA-staining dye DCV to isolate cells in specific phases of the cell cycle. DCV is a fluorescent dye with low cytotoxicity that enters living cells and selectively binds to DNA without fixation and permeabilization, thus, enabling the isolation of viable cells and assessment of their biological phenotypes (24). To the best of our knowledge, the present study is the first to isolate and characterize candidate quiescent CSCs in ESCC, thus, providing a novel target for investigating molecular mechanisms regulating quiescent CSCs and for developing novel therapeutic strategies.

Results of real-time PCR performed in the present study showed strong Nanog, BMI-1 and p63 expression in p75NTR-positive/G0-1 cells. Nanog is an embryonic pluripotency marker associated with the stem cell-like phenotype of CSCs (26). BMI-1 plays a crucial role in the self-renewal of CSCs in different cancers, including esophageal cancer $(27,28)$. p63 encoded by the oncogene TP63 regulates the growth and invasion of ESCC $(29,30)$.
These findings suggest that Nanog, BMI-1 and p63 regulate p75NTR-positive/G0-1 cells. NGF/proNGF/p75NTR axis plays a critical role in regulating the self-renewal of quiescent CSCs in breast cancer (31). Overexpression of NGF and its autocrine loop enhances the proliferation and migration of ESCC cell lines (32), suggesting that p75NTR signaling plays a role in the regulation of quiescent p75NTR-positive/ G0-1 cells.

In the present study, p75NTR-positive/G0-1 cells showed strong chemoresistance to CDDP. CDDP is one of the most effective anticancer drugs widely used in the first-line therapy of several cancers, including ESCC $(3,33)$. Because CDDP targets rapidly proliferating tumor cells (34), it generally does not affect quiescent CSCs. However, resistance to chemotherapies depends not only on cell quiescence but also on various molecular pathways in tumor cells (35). In this study, p75NTR-positive/G0-1 cells showed strong expression of ABCG2 and ERCC1. ABCG2 is an efflux transporter involved in multidrug resistance, and its overexpression is associated with response to chemotherapy and prognosis in ESCC (36). ERCC1 is involved in nucleotide excision repair pathway that modulates the efficacy of platinum-based chemotherapies by removing drug-induced DNA damage (37). In ESCC, expression of polymorphism in ERCC1 is a predictor of CDDP-based chemotherapy (38-40). Stem cell-related genes that were strongly expressed in p75NTR-positive/G0-1 cells, such as Nanog and BMI-1, are also involved in chemoresistance in different cancers (41-43). These findings strongly suggest that p75NTR-positive/G0-1 cells are responsible for resistance to CDDP-based chemotherapy in ESCC.

Immunohistochemical staining of surgically resected ESCC specimens showed p75NTR-positive ( $>5 \%$ of the tumor cells were stained) in $41.1 \%$ patients who showed favorable postoperative survival, which is consistent with the results of previous studies $(15,18)$. Moreover, p75NTR expression was significantly correlated with neoadjuvant chemotherapy, suggesting possible induction of p75NTR expression or selective survival of chemoresistant p75NTR-positive cells during chemotherapy. On the other hand, clinical significance of very small number of the cells with p75NTR expression (when $\leq 5 \%$ of the cancer cells were stained in a tumor) remains to be addressed. In addition, as was shown in a previous report, the expression of p75NTR decreases during progression of cancer in about half of ESCC tumors, resulting in complete loss of the expression in some cases (20). Therefore, other CSC markers are needed in tumors in which the expression of p75NTR is completely lost.

Double immunohistochemical staining of p75NTR-positive ESCC specimens detected p75NTR-positive/Ki-67-negative quiescent cells, with a median proportion of $13.2 \%$ cells (range, 3.0-80.1\%), which is consistent with the distribution of p75NTR-positive/Ki-67-negative KYSE cells both in vitro and in vivo. In addition, the proportion of p75NTR-positive/ Ki-67-negative cells was correlated with poorly differentiated histology and postoperative tumor recurrence, indicating that these cells represented relatively immature cells and are responsible for malignant potential in ESCC. Kaplan-Meier survival curves showed that patients with tumors having a high proportion of p75NTR-positive/Ki-67-negative cells showed poor survival; however, the correlation was not statistically 
significant. These results suggest that p75NTR-positive/Ki-67negative cells in ESCC possess quiescent CSC phenotypes similar to cultured KYSE cells. Further studies involving a large number of patients, extended follow-up, isolation of viable p75NTR-positive quiescent cells from fresh tumor samples may help elucidate the biological characteristics and clinical significance of quiescent CSCs.

In addition to p75NTR, CD44 (44) and CD90 (45) have been reported to be putative CSC markers in ESCC. In a previous report from our laboratory (18), the p75NTR-positive CD44negative fraction of KYSE-30 possessed CSC properties, such as stem cell-related gene expression, lower expression of differentiation markers, drug resistance and in vivo tumorigenicity. Furthermore, the majority of p75NTR-positive/CD44-negative cells were in a mitotically quiescent state, while p75NTR-positive/CD44-positive cells were actively proliferating. Similarly, the p75NTR-positive/CD90-negative subset was mitotically quiescent cells, while the p75NTR-positive/CD90-positive subset was actively proliferating. These observations indicate that p75NTR-positive/G0-1 fraction in this study is a specific subpopulation of ESCC with quiescent CSC properties, which cannot be identified using CD44 or CD90.

In conclusion, flow cytometric cell sorting based on p75NTR expression and cell cycle status helped in isolating and characterizing candidate quiescent CSCs from ESCC cell lines. These cells showed enhanced tumorigenicity and strong chemoresistance. Immunohistochemical examination detected small number of p75NTR-positive/Ki-67-negative cells in surgically resected ESCC specimens and indicate that these cells were associated with poorly differentiated histology of ESCC tumor. The results of the present study indicate that quiescent CSCs can be used as targets for investigating molecular process regulating quiescent CSC phenotypes and for developing novel therapeutic strategies.

\section{Acknowledgements}

The present study was supported by the Grant-in-Aid for Scientific Research (C) MEXT KAKENHI (grant nos. $15 \mathrm{~K} 10088$ and $15 \mathrm{~K} 10089)$. This study was also supported by the MEXT Translational Research Network Program (A-29).

\section{References}

1. Rice TW, Rusch VW, Apperson-Hansen C, Allen MS, Chen LQ, Hunter JG, Kesler KA, Law S, Lerut TE, Reed CE, et al: Worldwide esophageal cancer collaboration. Dis Esophagus 22: $1-8,2009$

2. Thallinger CM, Raderer M and Hejna M: Esophageal cancer: $A$ critical evaluation of systemic second-line therapy. J Clin Oncol 29: 4709-4714, 2011.

3. Ando N, Kato H, Igaki H, Shinoda M, Ozawa S, Shimizu H, Nakamura T, Yabusaki H, Aoyama N, Kurita A, et al: A randomized trial comparing postoperative adjuvant chemotherapy with cisplatin and 5-fluorouracil versus preoperative chemotherapy for localized advanced squamous cell carcinoma of the thoracic esophagus (JCOG9907). Ann Surg Oncol 19: 68-74, 2012.

4. Dalerba P and Clarke MF: Cancer stem cells and tumor metastasis: First steps into uncharted territory. Cell Stem Cell 1: 241-242, 2007

5. Clarke MF and Fuller M: Stem cells and cancer: Two faces of eve. Cell 124: 1111-1115, 2006

6. Reya $\mathrm{T}$ and Clevers $\mathrm{H}$ : Wnt signalling in stem cells and cancer Nature 434: 843-850, 2005.
7. D'Angelo RC and Wicha MS: Stem cells in normal development and cancer. Prog Mol Biol Transl Sci 95: 113-158, 2010.

8. Dean M, Fojo T and Bates S: Tumour stem cells and drug resistance. Nat Rev Cancer 5: 275-284, 2005.

9. Roesch A, Fukunaga-Kalabis M, Schmidt EC, Zabierowski SE Brafford PA, Vultur A, Basu D, Gimotty P, Vogt T and Herlyn M: A temporarily distinct subpopulation of slow-cycling melanoma cells is required for continuous tumor growth. Cell 141: 583-594, 2010.

10. Pece S, Tosoni D, Confalonieri S, Mazzarol G, Vecchi M, Ronzoni S, Bernard L, Viale G, Pelicci PG and Di Fiore PP: Biological and molecular heterogeneity of breast cancers correlates with their cancer stem cell content. Cell 140: 62-73, 2010.

11. Dembinski JL and Krauss S: Characterization and functional analysis of a slow cycling stem cell-like subpopulation in pancreas adenocarcinoma. Clin Exp Metastasis 26: 611-623, 2009.

12. Chen W, Dong J, Haiech J, Kilhoffer MC and Zeniou M: Cancer stem cell quiescence and plasticity as major challenges in cancer therapy. Stem Cells Int 2016: 1740936, 2016.

13. Adorno-Cruz V, Kibria G, Liu X, Doherty M, Junk DJ, Guan D, Hubert C, Venere M, Mulkearns-Hubert E, Sinyuk M, et al: Cancer stem cells: Targeting the roots of cancer, seeds of metastasis, and sources of therapy resistance. Cancer Res 75: 924-929, 2015.

14. Li L and Bhatia R: Stem cell quiescence. Clin Cancer Res 17: 4936-4941, 2011.

15. Okumura T, Tsunoda S, Mori Y, Ito T, Kikuchi K, Wang TC, Yasumoto S and Shimada Y: The biological role of the lowaffinity p75 neurotrophin receptor in esophageal squamous cell carcinoma. Clin Cancer Res 12: 5096-5103, 2006.

16. Huang SD, Yuan Y, Liu XH, Gong DJ, Bai CG, Wang F, Luo JH and Xu ZY: Self-renewal and chemotherapy resistance of p75NTR positive cells in esophageal squamous cell carcinomas. BMC Cancer 9: 9, 2009.

17. Li S, Yue D, Chen X, Wang L, Li J, Ping Y, Gao Q, Wang D, Zhang T, Li F, et al: Epigenetic regulation of CD271, a potential cancer stem cell marker associated with chemoresistance and metastatic capacity. Oncol Rep 33: 425-432, 2015.

18. Yamaguchi T, Okumura T, Hirano K, Watanabe T, Nagata T, Shimada Y and Tsukada K: p75 neurotrophin receptor expression is a characteristic of the mitotically quiescent cancer stem cell population present in esophageal squamous cell carcinoma. Int J Oncol 48: 1943-1954, 2016.

19. Okumura T, Shimada Y, Imamura M and Yasumoto S: Neurotrophin receptor p75(NTR) characterizes human esophageal keratinocyte stem cells in vitro. Oncogene 22: 4017-4026, 2003.

20. Okumura T, Shimada Y, Sakurai T, Hori R, Nagata T, Sakai Y and Tsukada K: Abnormal cell proliferation in the p75NTRpositive basal cell compartment of the esophageal epithelium during squamous carcinogenesis. Dis Esophagus 28: 634-643, 2015.

21. Shimada Y, Imamura M, Wagata T, Yamaguchi N and Tobe T: Characterization of 21 newly established esophageal cancer cell lines. Cancer 69: 277-284, 1992.

22. Li C, Heidt DG, Dalerba P, Burant CF, Zhang L, Adsay V, Wicha M, Clarke MF and Simeone DM: Identification of pancreatic cancer stem cells. Cancer Res 67: 1030-1037, 2007.

23. Scholzen T and Gerdes J: The Ki-67 protein: From the known and the unknown. J Cell Physiol 182: 311-322, 2000.

24. Bradford JA and Clarke ST: Dual-pulse labeling using 5-ethynyl2'-deoxyuridine (EdU) and 5-bromo-2'-deoxyuridine (BrdU) in flow cytometry. Curr Protoc Cytom 55: 7.38.1-7.38.15, 2011.

25. Hsu YC and Fuchs E: A family business: Stem cell progeny join the niche to regulate homeostasis. Nat Rev Mol Cell Biol 13: 103-114, 2012.

26. Hadjimichael C, Chanoumidou K, Papadopoulou N, Arampatzi P, Papamatheakis $\mathbf{J}$ and Kretsovali A: Common stemness regulators of embryonic and cancer stem cells. World J Stem Cells 7: 1150-1184, 2015.

27. Oren $\mathrm{O}$ and Smith BD: Eliminating cancer stem cells by targeting embryonic signaling pathways. Stem Cell Rev 13: 17-23, 2017.

28. Yu X, Jiang X, Li H, Guo L, Jiang W and Lu SH: miR-203 inhibits the proliferation and self-renewal of esophageal cancer stem-like cells by suppressing stem renewal factor Bmi-1. Stem Cells Dev 23: 576-585, 2014. 
29. Lee KB, Ye S, Park MH, Park BH, Lee JS and Kim SM: p63-Mediated activation of the $\beta$-catenin/c-Myc signaling pathway stimulates esophageal squamous carcinoma cell invasion and metastasis. Cancer Lett 353: 124-132, 2014.

30. Ye S, Lee KB, Park MH, Lee JS and Kim SM: p63 regulates growth of esophageal squamous carcinoma cells via the Akt signaling pathway. Int J Oncol 44: 2153-2159, 2014.

31. Tomellini E, Touil Y, Lagadec C, Julien S, Ostyn P, Ziental-Gelus N, Meignan S, Lengrand J, Adriaenssens E, Polakowska R, et al: Nerve growth factor and proNGF simultaneously promote symmetric self-renewal, quiescence, and epithelial to mesenchymal transition to enlarge the breast cancer stem cell compartment. Stem Cells 33: 342-353, 2015.

32. Tsunoda S, Okumura T, Ito T, Mori Y, Soma T, Watanabe G, Kaganoi J, Itami A, Sakai Y and Shimada Y: Significance of nerve growth factor overexpression and its autocrine loop in oesophageal squamous cell carcinoma. Br J Cancer 95: 322-330, 2006.

33. van Hagen P, Hulshof MC, van Lanschot JJ, Steyerberg EW, van Berge Henegouwen MI, Wijnhoven BP, Richel DJ, Nieuwenhuijzen GA, Hospers GA, Bonenkamp JJ, et al; CROSS Group: Preoperative chemoradiotherapy for esophageal or junctional cancer. N Engl J Med 366: 2074-2084, 2012.

34. Yuan L, Yu WM, Yuan Z, Haudenschild CC and Qu CK: Role of SHP-2 tyrosine phosphatase in the DNA damage-induced cell death response. J Biol Chem 278: 15208-15216, 2003.

35. Galluzzi L, Senovilla L, Zitvogel L and Kroemer G: The secret ally: Immunostimulation by anticancer drugs. Nat Rev Drug Discov 11: 215-233, 2012

36. Tsunoda S, Okumura T, Ito T, Kondo K, Ortiz C, Tanaka E, Watanabe G, Itami A, Sakai Y and Shimada Y: ABCG2 expression is an independent unfavorable prognostic factor in esophageal squamous cell carcinoma. Oncology 71: 251-258, 2006.

37. Furuta T, Ueda T, Aune G, Sarasin A, Kraemer KH and Pommier Y: Transcription-coupled nucleotide excision repair as a determinant of cisplatin sensitivity of human cells. Cancer Res 62: 4899-4902, 2002.
38. Huang J, Zhou Y, Zhang H, Qu T, Mao Y, Zhu H, Quan L, Xing P, Wang J, He J, et al: A phase II study of biweekly paclitaxel and cisplatin chemotherapy for recurrent or metastatic esophageal squamous cell carcinoma: ERCC1 expression predicts response to chemotherapy. Med Oncol 30: 343, 2013.

39. Chen WH, Xin PL, Pan QX, Chen YY, Wang CR, Zhang ZS, Chen YF, Zhang CY and Cai WJ: ERCC1 single nucleotide polymorphism C8092A, but not its expression is associated with survival of esophageal squamous cell carcinoma patients from Fujian province, China. PLoS One 9: e106600, 2014.

40. Yu X, Xiao H, Zhao B, Zhang X and Wang G: DNA repair gene ERCC1 C118T polymorphism predicts sensitivity of recurrent esophageal cancer to radiochemotherapy in a Chinese population. Thorac Cancer 6: 741-748, 2015.

41. Yang L, Zhang X, Zhang M, Zhang J, Sheng Y, Sun X, Chen Q and Wang LX: Increased Nanog expression promotes tumor development and Cisplatin resistance in human esophageal cancer cells. Cell Physiol Biochem 30: 943-952, 2012.

42. Yin T, Wei H, Leng Z, Yang Z, Gou S, Wu H, Zhao G, Hu X and Wang C: Bmi-1 promotes the chemoresistance, invasion and tumorigenesis of pancreatic cancer cells. Chemotherapy 57 : 488-496, 2011.

43. Banerjee Mustafi S, Chakraborty PK, Naz S, Dwivedi SK, Street M, Basak R, Yang D, Ding K, Mukherjee P and Bhattacharya R: MDR1 mediated chemoresistance: BMI1 and TIP60 in action. Biochim Biophys Acta 1859: 983-993, 2016.

44. Zhao JS, Li WJ, Ge D, Zhang PJ, Li JJ, Lu CL, Ji XD, Guan DX, Gao $\mathrm{H}, \mathrm{Xu} \mathrm{LY}$, et al: Tumor initiating cells in esophageal squamous cell carcinomas express high levels of CD44. PLoS One 6: e21419, 2011.

45. Tang KH, Dai YD, Tong M, Chan YP, Kwan PS, Fu L, Qin YR, Tsao SW, Lung HL, Lung ML, et al: A CD90+ tumor-initiating cell population with an aggressive signature and metastatic capacity in esophageal cancer. Cancer Res 73: 2322-2332, 2013. 